\title{
Core Ideas of the Application and Research of the Construction Technology of the Foundation Slab Concrete of the Building Foundation
}

\author{
Liu Zhi Jiao \\ Jiuquan Vocational and Technical College, Jiuquan 735000, Gansu
}

\begin{abstract}
Since our country entered the 21 st century, our country 's social economy has achieved remarkable achievements compared with the past, and the economic development has accelerated significantly. This has created good conditions for the healthy and sustainable development of the entire construction industry. Especially in recent years, the construction industry has occupied an increasingly important position. At present, with the advancement and development of science and technology, China's building technology has achieved better development than before. Various modern buildings have emerged continuously, and the construction of foundation slab concrete has emerged. With its own various characteristics, in the specific construction, it is necessary to strengthen the attention to various construction problems, such as easy cracking, large shrinkage and high water heating, etc. At the same time, it is necessary to combine specific construction standards and pay attention to research on related construction technology can promote the overall and steady improvement of the overall project quality.
\end{abstract}

\section{Introduction}

Under the influence of various factors in the actual building construction process, the application of building foundation concrete due to improper construction operations, temperature and other factors has increased the probability of crack problems and has a potential threat to the performance of the foundation floor structure. Affects the value of concrete after initial setting, and it will also cause corresponding changes due to internal and external forces. Concrete has a certain strength during the cold shrinking stage, which will constrain the internal coagulation greatly. At the same time, because concrete has the characteristics of thermal expansion and contraction, it will be affected by stress with the change of temperature. As a result, the application quality and structural performance of such structures are not guaranteed, which poses a potential threat to the stability of concrete structures. Therefore, when researching the construction of concrete foundation slabs, more attention should be paid to the introduction of its construction technology and scientific application, and the implementation of effective and effective construction plans to ensure the good construction conditions in this area.

\section{Introduction of Building Base Concrete}

\subsection{Reasonable Selection of Construction Materials}

The reliability of the application quality of concrete raw materials in building foundation construction is related to its structural stability. At the same time, because it is difficult to dissipate the hydration heat of the cement itself in the application of the foundation slab concrete structure, the internal temperature will rise accordingly, which will cause a large temperature difference with the outside stretching pressure. If the difference is obvious, the possibility of cracks is greater.

At the same time, when selecting fine aggregate, it must contain a certain amount of sand. The average particle size of medium sand is $0.5 \mathrm{~mm}$ larger than ordinary, and the mud content is controlled within 5\%. Compared with concrete with fine sand, the average average particle size of mixed concrete needs to reduce the amount of cement and water as much as possible, thereby contributing to the significant reduction of cement hydration heat and shrinkage performance. When selecting coarse aggregates, stones are classified well and large particle sizes usually need to be selected first. Generally, the particle size should be controlled between $5-25 \mathrm{~mm}$, and the mud content should not exceed $1 \%$. If concrete is mixed with crushed stone, it has good 
compressive strength itself, which can promote the significant reduction of the hydration heat of the cement itself and the significant increase of the concrete temperature [2].

In addition, when selecting materials, it is necessary to combine the relevant requirements of the specific construction scheme, strictly select the raw materials in accordance with the relevant requirements of the construction and the country, and fully guarantee the quality of the materials. Because concrete is mostly poured by pumping, in order to facilitate pumping, it is necessary to actively improve the workability of the concrete itself. An appropriate amount of fly ash can be added; strictly in accordance with the requirements of relevant specifications, the mixing of large-volume fly ash concrete The maximum limit for replacing cement with slag silicate is $25 \%$. Because fly ash is very committed to the improvement of concrete and workability and heat of hydration, but early addition will reduce the ultimate tensile value, which is not good for The impermeability and crack resistance of concrete need to be strictly controlled for this purpose (within 10\%). By using the admixture method, it can be calculated strictly in accordance with the requirements of the proportion on the basis of ensuring that the amount of cement in the proportion does not decrease dosage of fly ash. Regarding the admixture, the design lacks detailed regulations. Through analysis and comparison of other engineering experience, by placing $2 \mathrm{~kg}$ per cubic meter, the water reducing agent can help to significantly reduce the peak of hydrothermal heating and can effectively make up for concrete shrinkage. Promote the continuous improvement of its crack resistance. Before pouring, the concrete admixture dosage and usage performance should be reported to the construction unit in time [3].

\subsection{Reasonable Selection of Concrete Mix Ratio}

The design of the foundation slab concrete construction in related construction projects must conform to national requirements and technical standards. As for the determination of the concrete mix ratio, it is necessary to actively carry out the laboratory mix work. In order to ensure the timely supply of cement, it is necessary to actively measure and verify the heat of hydration of the cement to ensure that it can meet the relevant construction requirements. In the concrete construction process, it is necessary to make scientific and reasonable selection of materials. The optimization of the mixing ratio fully utilizes the "double admixture" technology, which can effectively control the rise rate of the internal temperature of the concrete and avoid or effectively delay the occurrence of temperature peaks. It can fully guarantee the quality of concrete and ensure its good working performance.

\section{Analysis of Construction Technology Points}

Because there are many procedures in the construction of the foundation slab concrete, and they are deeply restricted by various factors, during actual construction, it is necessary to carefully follow the actual construction situation of the project and find out the factors affecting the construction operation in a timely manner around the existing construction problems. In order to ensure construction quality and improve construction efficiency, we need to organically combine specific construction process requirements.

\subsection{Preparing for Construction}

Before construction, it is necessary to formulate a sound construction plan, to fully understand whether it meets the construction standards based on full discussion, to comprehensively examine various construction factors, such as personnel and materials. In addition, we need to fully understand the specific responsibilities that each construction staff should shoulder, and reasonably divide and arrange each staff's work. In addition, the materials such as plastic cloths and straw bags required for construction are carefully prepared, the surface of the base plate and the steel bars are marked carefully, and operations are strictly performed in accordance with the matters, so as to ensure the smooth development of various construction operations [4].

\subsection{Mixing and Supplying Concrete}

In order to fully ensure the temperature of the concrete, the concrete used for mixing the concrete must be placed in a shelter to prevent it from being exposed to the wind, the sun, and the rain. To ensure that it is always at a low temperature, it needs to be washed from time to time. Among them, when ice water and groundwater are used for washing, it is necessary to control the water temperature to not exceed $10 \%$, thereby helping to effectively reduce the temperature of the mixture. When mixing concrete, in order to avoid unnecessary measurement errors, the specific mixing and measurement of concrete can be fully aided by fully automatic microcomputers. When measuring, strictly follow certain standard values, such as 3\% gravel, $2 \%$ cement, $2 \%$ Water external additive. In particular, it should be noted that the mixing time should not be less than 2 minutes to effectively control the concrete transportation time. In order to fully grasp the pouring speed, ensure timely feedback of information and significantly reduce the waiting time of cement tankers, create good conditions for the normal supply of concrete, and avoid unnecessary cold joint problems caused by improper supply opportunities.

\subsection{Construction Technology}

Layered construction is used to pour the concrete. Regarding the pouring of concrete, the layered method can be used to fully ensure the strength of the concrete during construction, and ensure uniform rise. The specific principles must be strictly followed, that is, "thin layer pouring and step by step." During the actual pouring process, effective measures must be taken to 
prevent the cold joints that occur when the concrete flows naturally and the supply is slow. The concrete must be sufficiently cohesive, the slope must be controlled between 1:8, and the thickness of the slope layer must be $200-250 \mathrm{~mm}$. It is convenient for the upper concrete to cover the lower concrete before initial setting; for S-shaped pouring, the pouring temperature must be controlled within $28^{\circ} \mathrm{C}$, and the pouring belt swings in one direction.

When pumping concrete, a certain flow slope will inevitably be generated. Based on this, different vibrators need to be set in different areas. The first vibrator needs to be set at the concrete loading and unloading point to fully vibrate the concrete that has just come out of the pipe to ensure that it can fully flow to the bottom layer. The second time setting in the middle of the concrete can increase the compactness of the concrete. The third time is set at the bottom reinforcement, to fully shake the concrete flowing into the reinforcement to ensure that its compactness meets the basic requirements. In actual pouring, it is necessary to fully grasp the vibration time, and the speed cannot be too slow or too fast, and various operations are carried out strictly in accordance with the vibration sequence [5].

When pumping concrete, a certain amount of water must be generated, during which the concrete slurry will flow into the bottom of the pit along the foot of the slope. Therefore, a certain slope needs to be reserved during construction to ensure that the slurry water flows to the nearby pond, or is discharged outside the foundation pit with the help of a catchment pit, or the vibration direction is appropriately changed.

After the construction of the foundation slab concrete is completed, the surface of the slab used is thicker. Therefore, before the concrete is initially set, it is necessary for the construction unit to evenly crush $1-3 \mathrm{~cm}$ of crushed stone on the floor, and make full use of the vibration in real time. The vibrator can be scraped flat with a scraper; at the same time, two rubbing pressures are arranged after the initial setting, and the insulation material can be fully used when covering. Through such maintenance management, cracks can be effectively prevented from appearing on the concrete surface.

\subsection{Mastery of Scientific Temperature Control Methods}

The internal heat of concrete is difficult to fully dissipate during the construction process, and the external heat dissipation rate is relatively fast, especially during rain and at night, the temperature difference between the internal and external is large, so cracks are easily generated on the concrete surface. At the same time, after the concrete hardens, it will gradually shrink as the temperature decreases. Coupled with the influence of the foundation, the external restraining force will be greater, resulting in greater concrete strength, and eventually cracks will appear. In order to effectively circumvent the above situation, it is necessary to formulate a scientific temperature control scheme, organically combine the law of concrete hydration heat with the actual situation of the project, and strictly control the temperature difference of the concrete within $25^{\circ} \mathrm{C}$.

After the formulation of the scientific temperature control scheme is completed, effective control of different temperature measurement points needs to be realized. To this end, it is necessary to combine different thicknesses and vibration directions of the bottom plate. Within 3 hours after the completion of the vibrating operation, arrange a temperature measurement operation every hour, or after 6 hours, arrange a temperature measurement every 4 hours, until 10 hours after completing the vibrating operation. During this period, the temperature measurement results need to be carefully recorded. Once the temperature difference is greater than $22{ }^{\circ} \mathrm{C}$, it is necessary to report to the relevant departments in time and formulate scientific treatment measures. At present, XX-16 type thermometer is more commonly used in China, and its temperature probe uses $\mathrm{WZC}=010$ copper thermal resistance [6].

\subsection{Joint Construction}

Construction joints: After completing daily construction, a certain number of construction joints must be left at the construction site, usually at expansion joints and contraction joints.

Caulking: Sealing seams are usually carried out after the curing period has expired. Be careful to prevent unwanted debris, such as sediment, from entering the joints. Care must be taken to clean the debris in the joints before caulking, and then the fillers must be kept consistent at a high level with the board.

\subsection{Careful Construction of Concrete}

Compared with general building construction operations, there are obvious differences in concrete operations. After the construction is completed, the subsequent maintenance and management work must be done carefully to promote a significant extension of its service life. The specific work is as follows.

After finishing the pouring operation, it is necessary to carry out the surface compaction work carefully, and then cover the concrete surface with a straw bag, plastic cloth, etc., so as to obtain the ideal thermal insulation effect. At the same time, the hydration heat of the poured concrete will change rapidly during the construction process. To effectively avoid this, other materials, such as plastic cloth, can be covered. This effectively prevents unnecessary shrinkage problems caused by the evaporation of water on the concrete surface, and even cracks on the surface of the concrete, which can achieve dual effects, namely, heat preservation and moisture retention, and create certain conditions for the concrete surface to be constantly wet [7].

After the concrete construction is completed carefully, the maintenance work of concrete needs to continue. The main purpose is to carry out water conservation within half a month after the completion of the construction. Especially, it is necessary to focus on the maintenance pillars, wall-inserted steel bars, etc., to provide more 
solid coverage. Regarding the temperature measurement work, the specific situation must be agreed and confirmed by the relevant technical departments and personnel. After the temperature is constant, the plastic on the concrete surface layer must be removed one by one to fully ensure the effectiveness of concrete heat dissipation.

When carrying out the covering operation, if plastic film is used, it must be covered strictly and connected carefully. The concrete surface is strictly forbidden to be exposed outside, and straw bales, blankets, etc. need to be covered in special places such as elevators and water collecting wells.

\section{Conclusion}

Nowadays, our country's social economy has achieved rapid development compared with the previous period, thereby creating good conditions for the rapid development of construction technology, greatly expanding the scale of construction, and greatly increasing the number of large modern buildings. In addition, mass concrete construction projects have increased, but in the actual design, higher and higher requirements are imposed on the construction unit. Therefore, in the future, it is necessary to do a good job of building foundation concrete construction technology. To sum up, in order to better apply the construction technology of building foundation slab concrete, this article has specifically studied its core ideas in detail, and separately introduced the building foundation slab concrete and construction technology points.

\section{References}

1. Chen Kang. Research on winter construction technology of mass concrete for base plate of high-rise building [J]. Sichuan Cement, 2019 (08): 289.

2. Han $\mathrm{Xu}$. Research on construction technology of large-scale concrete in foundation engineering [J]. Journal of Shandong Agricultural Engineering College, 2019, 36 (02): 40-41.

3. Zeng Yizhuo. Construction technology of mass concrete for base plate of high-rise building [J]. Low Carbon World, 2018 (07): 184-185.

4. Tian Weiguo, Ling Huyan, Guan Congcong, Shao Peng, Hong Zhi. Construction technology of high-strength volume concrete in Block $\mathrm{C}$ of Hefei Evergrande Center [J]. Construction Technology, 2017, 46 (24): 1-7.

5. Ouyang Tingji. Discussion on the problems and preventive measures of water pouring in the floor concrete pouring in the deep foundation pit $[\mathrm{J}]$. Fujian Building Materials, 2016 (08): 104-105.

6. Shao Yuancai. Research on construction supervision of mass concrete for base plate of high-rise building [J]. Building Materials and Decoration, 2016 (34): 168-169.
7. Zhu Yimin, Gong Jian. Mass concrete construction technology for the base floor of the main building of Ningbo Global Shipping Plaza [J]. Construction and Construction, 2012,34 (01): 4-7. 